\title{
Magnetic Exchange in Spin Clusters
}

\author{
M. Georgiev* and H. Chamati \\ Institute of Solid State Physics, Bulgarian Academy of Sciences, Tsarigradsko Chaussée 72, 1784 Sofia, Bulgaria
}

(Dated: December 3, 2018)

\begin{abstract}
We investigate the role of exchange bridges in molecular magnets. We explore their effects on the distribution of the valence electrons and their contribution to the exchange processes. The present study is focused on a spin-half dimer with nonequivalent exchange bridges. Here, we derive an effective Hamiltonian that allows for an accurate estimation of the observables associated to the magnetic properties of the magnet. Our results are compared to those obtained by means of the conventional Heisenberg model that usually fails.
\end{abstract}

\section{INTRODUCTION}

Since decades the contribution of different bridging complexes to the magnetic properties of molecular magnets has motivated researchers to develop different approaches and give suggestions about the influence of bridging ions between neighboring magnetic centers. As the low-spin and short-bridged magnetic compounds are ideal candidates to study magneto-structural features, through the years, in many dimeric [1-3] and trimeric [4] compounds the nature of bridging structure is the subject of constant debate. One simple example is the $\mathrm{Cu}^{2+}$ cubane-type complex [5] with symmetric bridges described in the framework of a bilinear spin Hamiltonian. Further, the correlation of exchange constants with the structure parameters in alkoxo bridged cooper dimers was pointed out first in Ref. [5] and more recently in Ref. [6]. Additional efforts relating the bridging complexes and magnetism was shown in Ref. [7], where the interest in azido bridged complexes continues for decades [8-12]. Other prominent examples of magneto-structural effects are the complexes with Fe magnetic centers [13-16], the Ni based compounds [1720] and the Mn spin clusters [21-27].

In this paper we study the contribution of bridging structures to the exchange processes and its effect on the magnetic spectrum. In particular we show that complex bridging favors multiple exchange pathways between magnetic centers. To this end we discuss a spin-half dimer consisting of two non symmetric exchange bridges. We estimate the effect of both bridges and their contributions by deriving an effective Hamiltonian that allows for discrete exchange parameters accounting for the effects of bridging ions. The proposed Hamiltonian can be applied to a variety of molecular magnets with complex chemical environment and distortion in structure. Recently [28] we tested the proposed Hamiltonian by studying the magnetic spectra of trimeric compounds $\mathrm{A}_{3} \mathrm{Cu}_{3}\left(\mathrm{PO}_{4}\right)_{4}, \mathrm{~A}=(\mathrm{Ca}, \mathrm{Sr}$, $\mathrm{Pb}$ ) and the Nickel tetramer spin cluster $\mathrm{Ni}_{4} \mathrm{Mo}_{12}$.

\section{THE GENERIC HAMILTONIAN}

The generic Hamiltonian related to the electron-electron and electron-nuclei interactions in a magnetic cluster, within

\footnotetext{
*mgeorgiev@issp.bas.bg
}

the adiabatic approximation, reads

$$
\hat{H}=\sum_{i} \frac{\hat{p}_{i}^{2}}{2 \mathrm{~m}_{i}}+\sum_{\eta, i} \hat{U}\left(r_{\eta i}\right)+\frac{1}{2} \sum_{i \neq j} \hat{R}\left(r_{i j}\right),
$$

where $\hat{p}_{i}$ and $\mathrm{m}_{i}$ denotes the $i$-th electron momentum operator and mass, respectively. The potential energy operator $\hat{U}\left(r_{\eta i}\right)$ accounts for the interaction of the $i$-th electron with the $\eta$-th nucleus, separated by the distance $r_{\eta i}=\left|\mathbf{r}_{i}-\mathbf{R}_{\eta}\right|$ with $\mathbf{r}_{i}=\left(x_{i}, y_{i}, z_{i}\right)$ the coordinates of the $i$-th electron and $\mathbf{R}_{\eta}$ those of the $\eta$-th nuclei. The operator $\hat{R}\left(r_{i j}\right)$ is related to the electrostatic repulsion between $i$-th and $j$-th electrons over the distance $r_{i j}=\left|\mathbf{r}_{i}-\mathbf{r}_{j}\right|$. Obtaining the eigenstates of Eq. (2.1) assuming Coulomb potentials is a difficult task. Therefore, we estimate the transition energy associated to the exchange processes with the aid of the variational technique described in the following section.

\section{A. Molecular orbital approach}

For complex bondings we consider the approach of delocalized electrons developed within the framework of molecular orbital (MO) theory [29]. Within MO theory the electrons are not localized around the constituent nuclei, but are rather distributed over the entire molecule, thus occupying molecular orbitals. These orbitals are approximately given as a linear combination of the initial atomic orbitals (LCAOs) [30, 31]. Different constructions of molecular orbitals directly applied to study exchange processes in dimer complexes can be found in Ref. [1]. According to MO approach one distinguishes three categories of orbitals according to their contributions to the bonding energy and hence to the distribution of electrons. For further information on this topic we refer the interested reader to Refs. [32-37] and references therein.

In constructing the molecular orbitals we assume that only the valence orbitals of the nearest coupled ions overlap. Thus, we represent the $n$-th molecular orbital by the linear combination

$$
\phi_{n, m_{i}}\left(\mathbf{r}_{i}\right)=\sum_{\eta} c_{n}^{\eta} \psi_{\mu_{\eta}, m_{i}}^{\eta}\left(\mathbf{r}_{i}\right),
$$

where the real coefficients $c_{n}^{\eta}$ are functions of the overlap integrals between the directly coupled ions and the electronic eigenstates in a potential created by the $\eta$-th ion are given by

$$
\psi_{\mu_{\eta}, m_{i}}^{\eta}\left(\mathbf{r}_{i}\right)=\prod_{\alpha \in \mathbb{K}} \psi_{\mu_{\eta}}^{\eta}\left(\alpha_{i}\right)\left|m_{i}\right\rangle, \quad \mathbb{K}=\{x, y, z\},
$$


with $m_{i}$ the magnetic quantum number of the $i$-th electron. It is worth mentioning that for all $\eta$ and $i$ the functions in Eq. (2.3) are orthogonal an normalized such that

$$
\int \bar{\psi}_{\mu_{\eta}, m_{i}}^{\eta}\left(\mathbf{r}_{i}\right) \psi_{\mu_{\eta}^{\prime}, m_{i}^{\prime}}^{\eta}\left(\mathbf{r}_{i}\right) \mathrm{d} \mathbf{r}_{i}=\delta_{\mu_{\eta} \mu_{\eta}^{\prime}} \delta_{m_{i} m_{i}^{\prime}}
$$

and the overlap integral is

$$
0 \leq \int \bar{\psi}_{\mu_{\eta}, m_{i}}^{\eta}\left(\mathbf{r}_{i}\right) \psi_{\mu_{\eta^{\prime}}^{\prime}, m_{i}}^{\eta^{\prime}}\left(\mathbf{r}_{i}\right) \mathrm{d} \mathbf{r}_{i}<1
$$

Let us finally point out that the functions in Eq. (2.2) satisfy the orthogonality condition

$$
\int \bar{\phi}_{n, m_{i}}\left(\mathbf{r}_{i}\right) \phi_{n^{\prime}, m_{i}^{\prime}}\left(\mathbf{r}_{i}\right) \mathrm{d} \mathbf{r}_{i}=\delta_{n n^{\prime}} \delta_{m_{i} m_{i}^{\prime}}
$$

\section{B. Dimer state functions}

Consider $N$ valence electrons, where $N \geq 2$ is even. Let $n_{k}=N / 2+k$ be the number of highest in energy occupied molecular orbital, where $k \in \mathbb{N}_{0}$. As the transfer of electrons includes orbitals that are not fully occupied, in the spinhalf dimer magnets the corresponding process requires two orbitals. Therefore, knowing that $k$ is not fixed we assume these orbitals to be $N / 2+k$ and $N / 2+j$, where $k \neq j$. The remaining $(N-2) / 2$ molecular orbitals are fully occupied. As a consequence, we distinguish two sets of state functions. These states are obtained taking into account the Slater determinant $[38,39]$ and symmetrizing the corresponding functions according to the spin quantum numbers of each electron pair. The first set of states describe fully occupied molecular orbitals. These are

$$
\Phi_{S, M}^{n_{k}, n_{j}}\left(\mathbf{r}_{1}, \ldots, \mathbf{r}_{N}\right)=\sum_{P_{\mathbf{r}_{1}, \ldots r_{N}}} c_{\mathbf{r}_{N-1}, \mathbf{r}_{N}} \prod_{i}^{\frac{N}{2}-1} \frac{\Phi_{s_{2 i-1,2 i}}^{i}\left(\mathbf{r}_{2 i-1}, \mathbf{r}_{2 i}\right)}{\sqrt{2^{\frac{N}{2}} N !}} \Phi_{s_{N-1, N}}^{n_{k}, n_{j}}\left(\mathbf{r}_{N-1}, \mathbf{r}_{N}\right)|S, M\rangle,
$$

the second set describe a case with two half-filled orbitals. They read

$$
\Psi_{S, M}^{n_{k}, n_{j}}\left(\mathbf{r}_{1}, \ldots, \mathbf{r}_{N}\right)=\sum_{P_{\mathbf{r}_{1}, \ldots N_{N}}} c_{\mathbf{r}_{N-1}, \mathbf{r}_{N}} \prod_{i}^{\frac{N}{2}-1} \frac{\Phi_{s_{2 i-1,2 i}}^{i}\left(\mathbf{r}_{2 i-1}, \mathbf{r}_{2 i}\right)}{\sqrt{2^{\frac{N}{2}} N !}} \Psi_{s_{N-1, N}}^{n_{k}, n_{j}}\left(\mathbf{r}_{N-1}, \mathbf{r}_{N}\right)|S, M\rangle,
$$

where the sum runs over all permutations on the set of coordinates $\mathbf{r}_{1} \ldots \mathbf{r}_{N}$ and the functions in the summands are

$$
\begin{gathered}
\Phi_{s_{2 i-1,2 i}^{i}}^{i}\left(\mathbf{r}_{2 i-1}, \mathbf{r}_{2 i}\right)=\frac{1}{2}\left[\phi_{i}\left(\mathbf{r}_{2 i-1}\right) \phi_{i}\left(\mathbf{r}_{2 i}\right)-(-1)^{1+s_{2 i-1,2 i}} \phi_{i}\left(\mathbf{r}_{2 i-1}\right) \phi_{i}\left(\mathbf{r}_{2 i}\right)\right], \\
\Phi_{s_{N-1, N}}^{n_{k}, n_{j}}\left(\mathbf{r}_{N-1}, \mathbf{r}_{N}\right)=\frac{1}{\sqrt{2}}\left[\phi_{n_{k}}\left(\mathbf{r}_{N-1}\right) \phi_{n_{k}}\left(\mathbf{r}_{N}\right)-(-1)^{1+s_{N-1, N}} \phi_{n_{j}}\left(\mathbf{r}_{N-1}\right) \phi_{n_{j}}\left(\mathbf{r}_{N}\right)\right]
\end{gathered}
$$

and

$$
\Psi_{s_{N-1, N}}^{n_{k}, n_{j}}\left(\mathbf{r}_{N-1}, \mathbf{r}_{N}\right)=\frac{1}{\sqrt{2}}\left[\phi_{n_{k}}\left(\mathbf{r}_{N-1}\right) \phi_{n_{j}}\left(\mathbf{r}_{N}\right)-(-1)^{1+s_{N-1, N}} \phi_{n_{j}}\left(\mathbf{r}_{N-1}\right) \phi_{n_{k}}\left(\mathbf{r}_{N}\right)\right]
$$

Moreover, for $i, j=1, \ldots, N$ the permutation coefficients

$$
c_{\mathbf{r}_{i}, \mathbf{r}_{j}}=(-1)^{i+j+1}, \quad c_{\mathbf{r}_{j}, \mathbf{r}_{i}}=-(-1)^{1+s_{N-1, N}}(-1)^{i+j+1},
$$

account for the antisymmetry of triplet function in Eq. $(2.7 \mathrm{c})$. The spin part in the Eq. (2.6a) and (2.6b) is given by

$$
|S, M\rangle=\otimes_{i=1}^{\frac{N}{2}}\left|s_{2 i-1,2 i}, m_{2 i-1,2 i}\right\rangle \text {. }
$$

Notice that $S$ and $M$ are not the total spin and magnetic quantum numbers of the system. The latter represent the sets of all spin and magnetic quantum numbers for each pair of electrons, respectively. Since we study a system with two effective spinhalf centers one has to bear in mind the following constraints

$$
\sum_{i=1}^{N / 2} \hat{\mathbf{s}}_{2 i-1,2 i}^{2}|S, M\rangle=S(S+1)|S, M\rangle
$$

$$
\sum_{i=1}^{N / 2} \hat{s}_{2 i-1,2 i}^{z}|S, M\rangle=M|S, M\rangle
$$

with $S=0,1$ and $M=0, \pm 1$. We would like to point out that with respect to Eq. (2.5) for all $k$ and $j$ the functions in Eq. (2.6a) and (2.6b) are mutually orthogonal. Furthermore, they have to be used by having in mind that for $N=2$ their spatial parts reduce to Eq. (2.7b) and (2.7c), respectively.

\section{Key integrals}

Hamiltonian in Eq. (2.1) accounts for the kinetic and repulsion energies of all electrons in the system. As the $N / 2$ lower orbitals are fully occupied it is therefore sufficient to calculate 
the corresponding average energy value. Then we are left only with the operators related to orbitals that are not fully occupied. Similar to the Hartree method $[40,41]$ this procedure will demonstrate explicitly the contribution of $(N / 2+k)$-th orbitals to the exchange processes. Hence, with $N-2$ electrons, occupying all molecular orbitals lower in energy than the $(N / 2+k)$-th orbital, we have

$$
\hat{H}^{\prime}=\prod_{i, j}^{\frac{N}{2}-1} \int \ldots \int \bar{\Phi}_{0}^{j}\left(\mathbf{r}_{2 j-1}, \mathbf{r}_{2 j}\right) \hat{H} \Phi_{0}^{i}\left(\mathbf{r}_{2 i-1}, \mathbf{r}_{2 i}\right) \mathrm{d} \mathbf{r}_{1} \ldots \mathrm{d} \mathbf{r}_{N-2}
$$

Consequently, we distinguish four types of integrals related with the processes of exchange and transfer of electrons. Considering the $(N / 2+k)$-th orbital we obtain the integral

$$
U_{n_{k}}=\iint \bar{\phi}_{n_{k}}\left(\mathbf{r}_{N-1}\right) \bar{\phi}_{n_{k}}\left(\mathbf{r}_{N}\right) \hat{H}^{\prime} \phi_{n_{k}}\left(\mathbf{r}_{N-1}\right) \phi_{n_{k}}\left(\mathbf{r}_{N}\right) \mathrm{d} \mathbf{r}_{N-1} \mathrm{~d} \mathbf{r}_{N}
$$

accounting for the kinetic and potential energies of two electrons occupying the same orbital. The hopping integral

$t_{n_{k}}=\iint \bar{\phi}_{n_{k}}\left(\mathbf{r}_{N-1}\right) \bar{\phi}_{n_{k}}\left(\mathbf{r}_{N}\right) \hat{H}^{\prime} \phi_{n_{k}}\left(\mathbf{r}_{N-1}\right) \phi_{n_{j}}\left(\mathbf{r}_{N}\right) \mathrm{d} \mathbf{r}_{N-1} \mathrm{~d} \mathbf{r}_{N}$, associated with the transfer of an electron between two orbitals. The integral

$V_{n_{k} n_{j}}=\iint \bar{\phi}_{n_{k}}\left(\mathbf{r}_{N-1}\right) \bar{\phi}_{n_{j}}\left(\mathbf{r}_{N}\right) \hat{H}^{\prime} \phi_{n_{k}}\left(\mathbf{r}_{N-1}\right) \phi_{n_{j}}\left(\mathbf{r}_{N}\right) \mathrm{d} \mathbf{r}_{N-1} \mathrm{~d} \mathbf{r}_{N}$,

representing the energy of two electrons occupying different orbitals and the exchange integral

$D_{n_{k} n_{j}}=\iint \bar{\phi}_{n_{k}}\left(\mathbf{r}_{N-1}\right) \bar{\phi}_{n_{j}}\left(\mathbf{r}_{N}\right) \hat{H}^{\prime} \phi_{n_{j}}\left(\mathbf{r}_{N-1}\right) \phi_{n_{k}}\left(\mathbf{r}_{N}\right) \mathrm{d} \mathbf{r}_{N-1} \mathrm{~d} \mathbf{r}_{N}$,

associated with the energy of direct exchange of two electrons between orbitals $n_{k}$ and $n_{j}$.

The integrals in Eq. (2.9a) and (2.9b) are nonzero only when the electron's spins are antiparallel, see Eq. (2.7b). By analogy with a closed shell system the single orbital term describes a case with compactly occupied molecular orbitals, i.e. a nonmagnetic molecule. Therefore, Eq. (2.9a) and (2.9b) favor antiferromagnetism and refer to magnetic insulators. If according to the Hund's rule a triplet state related to any two electrons occupying orbitals $N / 2+k$ and $N / 2+j$, respectively, is the ground state, then the integrals in Eq. (2.9c) and (2.9d) will determine the values of the transition energy. Therefore, taking into account Eq. (2.8) and (2.9) we obtain the following relations

$$
\begin{aligned}
& \int \ldots \int \bar{\Psi}_{1, M}^{n_{k}, n_{j}}\left(\mathbf{r}_{1}, \ldots, \mathbf{r}_{N}\right) \hat{H} \Psi_{1, M}^{n_{k^{\prime}, n_{j^{\prime}}}}\left(\mathbf{r}_{1}, \ldots, \mathbf{r}_{N}\right) \mathrm{d} \mathbf{r}_{1} \ldots \mathrm{d} \mathbf{r}_{N}=\left(V_{n_{k} n_{j}}-D_{n_{k} n_{j}}\right) \delta_{k k^{\prime}} \delta_{j j^{\prime}}+\mathscr{O}\left(\frac{1}{\sqrt{2^{N-2} N !}}\right), \\
& \int \ldots \int \bar{\Psi}_{0,0}^{n_{k}, n_{j}}\left(\mathbf{r}_{1}, \ldots, \mathbf{r}_{N}\right) \hat{H} \Psi_{0,0}^{n_{k^{\prime}}, n_{j^{\prime}}}\left(\mathbf{r}_{1}, \ldots, \mathbf{r}_{N}\right) \mathrm{d} \mathbf{r}_{1} \ldots \mathrm{d} \mathbf{r}_{N}=\left(V_{n_{k} n_{j}}+D_{n_{k} n_{j}}\right) \delta_{k k^{\prime}} \delta_{j j^{\prime}}+\mathscr{O}\left(\frac{1}{\sqrt{2^{N-2} N !}}\right), \\
& \int \ldots \int \bar{\Phi}_{0,0}^{n_{k}, n_{j}}\left(\mathbf{r}_{1}, \ldots, \mathbf{r}_{N}\right) \hat{H} \Phi_{0,0}^{n_{k^{\prime}}, n_{j^{\prime}}}\left(\mathbf{r}_{1}, \ldots, \mathbf{r}_{N}\right) \mathrm{d} \mathbf{r}_{1} \ldots \mathrm{d} \mathbf{r}_{N}=\left(U_{n_{k} n_{j}}+D_{n_{k} n_{j}}\right) \delta_{k k^{\prime}} \delta_{j j^{\prime}}+\mathscr{O}\left(\frac{1}{\sqrt{2^{N-2} N !}}\right)
\end{aligned}
$$

and

$$
\int \ldots \int \bar{\Psi}_{0,0}^{n_{k}, n_{j}}\left(\mathbf{r}_{1}, \ldots, \mathbf{r}_{N}\right) \hat{H} \Phi_{0,0}^{n_{k^{\prime}}, n_{j^{\prime}}}\left(\mathbf{r}_{1}, \ldots, \mathbf{r}_{N}\right) \mathrm{d} \mathbf{r}_{1} \ldots \mathrm{d} \mathbf{r}_{N}=2 t_{n_{k} n_{j}} \delta_{k k^{\prime}} \delta_{j j^{\prime}}+\mathscr{O}\left(\frac{1}{\sqrt{2^{N-2}} N !}\right)
$$

where

$$
t_{n_{k} n_{j}}=\frac{1}{2}\left(t_{n_{k}}+t_{n_{j}}\right), \quad U_{n_{k} n_{j}}=\frac{1}{2}\left(U_{n_{k}}+U_{n_{j}}\right) .
$$

Notice that the integrals in Eq. (2.11) vanish rapidly with $N$ for $k^{\prime} \neq k$ and $j^{\prime} \neq j$. Therefore, in terms of matrices one can reduce the column and row number of the corresponding to Eq. (2.11) matrix into a $(5 \times 5)$ and represent it by the sum $3 \oplus 2$. The $(3 \times 3)$ matrix include the energy associated with the triplet group in Eq. $(2.10)$ and the $(2 \times 2)$ one represent the energy related with the singlet states in Eq. (2.11). Accordingly, the set of all eigenstates consists of the triplet group represented by the functions in Eq. (2.10) and the singlet group

$$
\begin{aligned}
\Omega_{0,0}^{n_{k}, n_{j}}\left(\mathbf{r}_{1}, \ldots, \mathbf{r}_{N}\right)= & \frac{\sin \phi}{\sqrt{2}} \Phi_{0,0}^{n_{k}, n_{j}}\left(\mathbf{r}_{1}, \ldots, \mathbf{r}_{N}\right) \\
& +\frac{\cos \phi}{\sqrt{2}} \Psi_{0,0}^{n_{k}, n_{j}}\left(\mathbf{r}_{1}, \ldots, \mathbf{r}_{N}\right)
\end{aligned}
$$




$$
\begin{aligned}
\Theta_{0,0}^{n_{k}, n_{j}}\left(\mathbf{r}_{1}, \ldots, \mathbf{r}_{N}\right)= & \frac{\cos \phi}{\sqrt{2}} \Phi_{0,0}^{n_{k}, n_{j}}\left(\mathbf{r}_{1}, \ldots, \mathbf{r}_{N}\right) \\
& -\frac{\sin \phi}{\sqrt{2}} \Psi_{0,0}^{n_{k}, n_{j}}\left(\mathbf{r}_{1}, \ldots, \mathbf{r}_{N}\right)
\end{aligned}
$$

where

$$
\phi=\arctan \left(\frac{4 t_{n_{k} n_{j}}}{U_{n_{k} n_{j}}-V_{n_{k} n_{j}}+\sqrt{16 t_{n_{k} n_{j}}^{2}+\left(U_{n_{k} n_{j}}-V_{n_{k} n_{j}}\right)^{2}}}\right) .
$$

From now on we consider only the state in Eq. (2.12a) since it correspond to the lower energy value for all $t_{n_{k} n_{j}}$.

\section{MULTIPLE EXCHANGE PATHWAYS}

For isolated diatomic and triatomic magnetic units the exchange pathways are unique. In general, the GoodenoughKanamori-Anderson rules [21, 42-44] holds and the magnetic spectra are usually explained in terms of the Heisenberg model [45]. With respect to the nature of ligands in some magnetic compounds, anisotropic spin Hamiltonians are valuable for studying the magnetic properties. Further, in the case of mono or diatomic intermediate bridges in periodic latices the competition between kinetic energy and Coulomb repulsion can be adequately studied within the framework of the Hubbard model [46, 47]. However, in complex molecular magnets, the exchange process between two effective magnetic centers involves a number of intermediate nonmagnetic ions. Thus, if two such centers are connected by more than one intricate bridge, see for example the molecular magnet $\mathrm{Ni}_{4} \mathrm{MO}_{12}$ [48], it is possible to have more than one energetically favorable distribution of unpaired valence electrons. Accordingly, multiple independent magnetic excitations that do not arise due to the anisotropy related with spin-orbit coupling nor to the existence of electronic bands, but rather results from the activation of different exchange bridges, will emerge. A sign for the absence of a unique exchange bridge can be the broadened excitation peaks in the observed magnetic spectrum and the enhanced response of the molecular magnet to an external magnetic field. In particular since the values of the overlap integrals depend on the angles between coupled ions the effect of applied magnetic field or changes in temperature may cause variations in the energy of the considered molecular orbitals altering the distribution of valence electrons and hence the values of the transition energy.

\section{A. The effective Hamiltonian}

In order to simplify all further expressions we change the notations by setting $\tau=\left(n_{k}, n_{j}\right)$ as a general index denoting both the half filled molecular orbitals and the number of valence electrons. Therefore, as the functions in Eq. (2.6) correspond to a certain distribution of electrons, $\tau$ will indicates all existing exchange bridges. Then, for $\tau^{\prime} \neq \tau$ one has different number of electrons $N^{\prime} \neq N$. Further, as the number of electrons is related to the number of all spin pairs one has $S^{\prime} \neq S$ and $M^{\prime} \neq M$. Then, in order to address the aforementioned assumptions correctly we label the number of valence electrons $N$ and the sets of spin and magnetic quantum numbers $S$ and $M$, according to the corresponding bridge obtaining $N_{\tau}$, $S_{\tau}$ and $M_{\tau}$, respectively. Hamiltonian in Eq. (2.1) depends on the number of valence electrons and coupled ions. Henceforward we will be denoting the later by $\hat{H}_{\tau}$.

Since the unpaired electrons can be distributed over any of the considered bridges, the generic state functions will account for all probabilities. Thus, setting $\mathbf{r}_{\tau}=\left\{\mathbf{r}_{1}, \ldots, \mathbf{r}_{N_{\tau}}\right\}$ and using the states in Eq. (2.6a), (2.6b) and (2.12a), within the current notations we obtain the triplet states

$$
\Psi_{1, m}\left(\mathbf{r}_{\tau}, \ldots, \mathbf{r}_{\tau^{\prime}}\right)=\sum_{\tau} c_{1}^{\tau} \Psi_{1, M_{\tau}}^{\tau}\left(\mathbf{r}_{\tau}\right), \quad \forall S_{\tau}=1
$$

and the singlet state

$$
\Psi_{0,0}\left(\mathbf{r}_{\tau}, \ldots, \mathbf{r}_{\tau^{\prime}}\right)=\sum_{\tau} c_{0}^{\tau} \Omega_{0,0}^{\tau}\left(\mathbf{r}_{\tau}\right), \quad \forall S_{\tau}=0
$$

where for all $\tau$ the quantities $s=S_{\tau}, m=M_{\tau}$ are the dimer effective spin and magnetic quantum numbers. The last functions are orthonormal and the coefficients $c_{S_{-}}^{\tau} \in \mathbb{R}$ depend on the spin quantum numbers since for the singlet state, $S_{\tau}=0$, the two electrons are closer to each other than in the case of triplet states, $S_{\tau}=1$.

We would like to point out that according to the direct sum of the spin subspaces of the exchange pathways one has $\left\langle S_{\tau}, M_{\tau} \mid S_{\tau^{\prime}}, M_{\tau^{\prime}}\right\rangle=\delta_{\tau \tau^{\prime}}$. Notice also that for all $\tau$ the constraints in Eq. (2.8) are always satisfied.

Taking into consideration the integrals in Eq. (2.10) and (2.11) for all $\tau$ the energy expectation values read

$$
\begin{aligned}
& E_{1, M_{\tau}}^{\tau}=\frac{\int \bar{\Psi}_{1, M_{\tau}}^{\tau}\left(\mathbf{r}_{\tau}\right) \hat{H}_{\tau} \Psi_{1, M_{\tau}}^{\tau}\left(\mathbf{r}_{\tau}\right) \mathrm{d} \mathbf{r}_{\tau}}{\int \bar{\Psi}_{1, M_{\tau}}^{\tau}\left(\mathbf{r}_{\tau}\right) \Psi_{1, M_{\tau}}^{\tau}\left(\mathbf{r}_{\tau}\right) \mathrm{d} \mathbf{r}_{\tau}}, \\
& E_{0,0}^{\tau}=\frac{\int \bar{\Omega}_{0,0}^{\tau}\left(\mathbf{r}_{\tau}\right) \hat{H}_{\tau} \Omega_{0,0}^{\tau}\left(\mathbf{r}_{\tau}\right) \mathrm{d} \mathbf{r}_{\tau}}{\int \bar{\Omega}_{0,0}^{\tau}\left(\mathbf{r}_{\tau}\right) \Omega_{0,0}^{\tau}\left(\mathbf{r}_{\tau}\right) \mathrm{d} \mathbf{r}_{\tau}},
\end{aligned}
$$

where $\mathrm{d} \mathbf{r}_{\tau}=\mathrm{d} \mathbf{r}_{1} \ldots \mathrm{d} \mathbf{r}_{N_{\tau}}$. In terms of the integrals in Eq. (2.9), the energy values in Eq. (3.3) are given by

$$
E_{1, M_{\tau}}^{\tau}=V_{\tau}-D_{\tau}, \quad M_{\tau}=0, \pm 1
$$

$$
E_{0,0}^{\tau}=D_{\tau}+\frac{U_{\tau}+V_{\tau}}{2}-\sqrt{4 t_{\tau}^{2}+\frac{\left(U_{\tau}-V_{\tau}\right)^{2}}{4}}
$$

respectively. Therefore, the expectation values of the Hamiltonian in Eq. (2.1) obtained from states written in Eq. (3.1) and (3.2) can then be written as

$$
E_{s, m}=\sum_{\tau}\left|c_{S_{\tau}}^{\tau}\right|^{2} E_{S_{\tau}, M_{\tau}}^{\tau}
$$


Notice that the Hamiltonians related with the different bridging structures commute, $\left[\hat{H}_{\tau}, \hat{H}_{\tau^{\prime}}\right]=0$. The last energy values represent the spectrum of an effective Hamiltonian satisfying

$$
\hat{H}_{e f f}|s, m\rangle=E_{s, m}|s, m\rangle,
$$

where the states $|s, m\rangle$ are eigenstates of the effective dimer total spin operator $\hat{\mathbf{s}}=\left(\hat{s}^{x}, \hat{s}^{y}, \hat{s}^{z}\right)$, with

$$
\hat{\mathbf{s}}^{2}|s, m\rangle=s(s+1)|s, m\rangle, \quad \hat{s}^{z}|s, m\rangle=m|s, m\rangle .
$$

With respect to the spin quantum number $s$ the Hamiltonian is $(2 s+1)$-fold degenerate and hence the considered system allows only one transition, $\left|E_{1, m}-E_{0,0}\right|$. On the other hand, as the energy values in Eq. (3.5) depend on the probability coefficients in Eq. (3.1) and (3.2), multiple transitions related to the spatial part of the state functions are allowed. However, relying on such assumptions, one has to take into account that the energy conservation law do not allow the simultaneous existence of more than one excitation. Thereby, for the transition energy we obtain

$$
|\Delta E|=\left.\sum_{\tau}|| c_{1}^{\tau}\right|^{2} E_{1, M_{\tau}}^{\tau}-\left|c_{0}^{\tau}\right|^{2} E_{0,0}^{\tau} \mid .
$$

Although the coefficients in Eq. (3.7) can be represented by analytical functions one can observe a number of different values for $\Delta E$ related with a discrete spectrum. This follows from the independence of all possible exchange pathways and the difference of the electron's behavior at triplet and singlet states. As a consequence, for $s=0$ and at certain conditions the electrons could be localized only on one of the exchange bridges. In contrary, for the triplet state both electrons could be distributed over all bridges.

\section{B. The simplest case}

Assuming a molecule with unique exchange bridge of one or two intermediate atoms the sum in Eq. (3.7) is reduced to a single term with $\left|c_{1}^{\tau}\right|^{2}=1$ and $\left|c_{0}^{\tau}\right|^{2}=1$. Consequently, suggesting an antiferromagnetic ground state and taking into account Eq. (3.4), for the transition energy in Eq. (3.7) we have

$$
\Delta E=-2 D_{\tau}-\frac{1}{2}\left(U_{\tau}+V_{\tau}\right)+\frac{1}{2} \sqrt{16 t_{\tau}^{2}+\left(U_{\tau}-V_{\tau}\right)^{2}} .
$$

Within the selected ground state the value of integral in Eq. (2.9c) could be considered as rather smaller than the single orbital integral in Eq. (2.9a) and one can use of the inequality $U_{\tau} \gg V_{\tau}$. Furthermore, since the intermediate and magnetic ions are of different kind, their mutual orientation renders the value of corresponding overlap integral negligible. This implies that $U_{\tau}>t_{\tau}$ and $t_{\tau}>D_{\tau}$, see Eq. (2.9). Perturbing over $t_{\tau} / U_{\tau}$ and taking into account only the first two terms from the series, we obtain

$$
\Delta E=\frac{4 t_{\tau}^{2}}{U_{\tau}}-2 D_{\tau} .
$$

For some compounds the direct exchange term $D_{\tau}$ is assumed negligible. The ground state is antiferromagnetic and the system behaves as a magnetic insulator, since $t_{\tau}$ and $U_{\tau}$ favor antifferomagnetism, see Eq. (2.6a) and (2.7b). A different approach describing localized electrons by using Wannier functions [49] and leading to analogous conclusions are introduced in Ref. [50].

\section{THE SPIN HAMILTONIAN}

Within the spin space, the magnetic excitation energy is associated only with molecular orbitals that are not fully occupied. Therefore, the total spin of the chemical complex is effectively taken into account by considering the number of unpaired electrons. Then, all magnetic features are interpreted in terms of either effective or fictitious spins of magnetic centers usually representing transition metal ions.

Operating with a conventional bilinear spin Hamiltonian, one won't be able to associate each of the excitations in Eq. (3.7) within a single singlet-triplet transition. Therefore, we pursue a different approach to account for the features arising from the existence of multiple exchange pathways.

Let $i=1,2$ indicate both effective magnetic centers in the considered spin dimer and $\hat{\mathbf{s}}_{i}=\left(\hat{s}_{i}^{\alpha}\right)_{\alpha \in \mathbb{K}}$ are their corresponding spin operators with

$$
\hat{\mathbf{s}}_{i}^{2}\left|s_{i}, m_{i}\right\rangle=s_{i}\left(s_{i}+1\right)\left|s_{i}, m_{i}\right\rangle, \quad \hat{s}_{i}^{z}\left|s_{i}, m_{i}\right\rangle=m_{i}\left|s_{i}, m_{i}\right\rangle,
$$

where $s_{i}$ and $m_{i}$ are the respective spin and magnetic quantum numbers. Notice that in fact the last spin operators account for the spins of both unpaired electrons. Further, let $\hat{\mathbf{s}}=\hat{\mathbf{s}}_{1}+\hat{\mathbf{s}}_{2}$ be the total spin operator written in Eq. (3.6). In order to obtain an energy spectrum consistent with the transitions in Eq. (3.7) we propose the following Hamiltonian

$$
\hat{\mathscr{H}}=J\left(\hat{\mathbf{s}}_{1} \cdot \hat{\boldsymbol{\sigma}}_{2}+\hat{\mathbf{s}}_{2} \cdot \hat{\boldsymbol{\sigma}}_{1}\right)-g \mu_{\mathrm{B}} \hat{s}^{z} B,
$$

where $J$ is an effective constant, $g$ is the isotropic $g$-tensor, $\mu_{\mathrm{B}}$ is the Bohr magneton, $B$ represents the externally applied magnetic field and the operator $\hat{\boldsymbol{\sigma}}_{i}=\left(\hat{\sigma}_{i}^{x}, \hat{\sigma}_{i}^{y}, \hat{\sigma}_{i}^{z}\right)$ effectively accounts for the differences in valence electron's distribution with respect to the $i$-th magnetic center. We would like to point out that the atomic orbitals of selected magnetic centers are considered as quenched and in accordance with the proposed superposition in Eq. (2.2) the $g$-tensor does not alter.

Since the excitation energy is related with the transition between singlet and triplet states of the total dimer spin space one has to account for the total $\sigma$-operator defined by

$$
\hat{\sigma}^{\alpha}|s, m\rangle=a_{s, m} \hat{s}^{\alpha}|s, m\rangle,
$$

where $a_{s, m} \in \mathbb{R}$. As the spins of both magnetic centers are paired their relevant $\sigma$-operators share the coefficient in Eq. (4.2) and govern the transformations

$$
\hat{\sigma}_{i}^{\alpha}|s, m\rangle=a_{s, m} \hat{s}_{i}^{\alpha}|s, m\rangle, \quad i=1,2 .
$$

The rising and lowering $\sigma$-operators corresponding to Eq. (4.2) satisfy

$$
\hat{\sigma}^{ \pm}|s, m\rangle=a_{s, m} \hat{s}^{ \pm}|s, m\rangle,
$$


where $\hat{s}^{ \pm}$are the total rising and lowering spin operators. Thereby, taking into consideration Eq. (3.6), (4.2), (4.4) and the following three cases $m=s,-s<m<s$ and $m=-s$, where $s \neq 0$ for the eigenvalues of total sigma square operator we have

$$
\begin{gathered}
a_{s, s}^{2} s^{2}+a_{s, s} a_{s, s-1} s, \\
\frac{1}{2} a_{s, m}\left[a_{s, m+1}+a_{s, m-1}\right] s(s+1)+a_{s, m}^{2} m^{2} \\
-\frac{1}{2} a_{s, m} m\left[a_{s, m+1}(m+1)+a_{s, m-1}(m-1)\right], \\
a_{s,-s}^{2} s^{2}+a_{s,-s} a_{s, 1-s} s .
\end{gathered}
$$

The eigenvalues of $\sigma^{z}$ can be obtained directly from Eq. (4.2).

All excitations are a result of the transition between singlet and triplet states, see Eq. (3.7), where the total spin and magnetic quantum numbers are always preserved, see also Eq. (2.8). Therefore, we imply the following constraints

$$
\begin{gathered}
\hat{\sigma}^{z}|s, m\rangle=h_{s} m|s, m\rangle, \\
\hat{\boldsymbol{\sigma}}^{2}|s, m\rangle=h_{s}^{2} s(s+1)|s, m\rangle,
\end{gathered}
$$

where the parameters $h_{s}$ account for the changes of electrons distributions in the molecule and thus the variation of all coefficients in Eq. (2.2), (3.1) and (3.2) due to the action of B. For $B=0$ and all $s$ we have $h_{s}=1$.

Within the last constraints one distinguish three cases:

(1) $s \neq 0$ and $m \neq 0$, then

$$
a_{s, m \pm 1}=a_{s, m}=h_{s} .
$$

As a consequence, for $B=0$, the $\sigma$-operator transforms the spin eigenstates as the spin operator does and the Hamiltonian in Eq. (4.1) coincide with its Heisenberg counterpart.

(2) $s \neq 0$ and $m=0$, then the coefficients in Eq. (4.2) are restricted only by the Eq. (4.6b). As a result, from Eq. (4.6a), (4.5b) and (4.6b) one obtains

$$
a_{s, \pm 1}=a_{s, 0}= \pm h_{s}
$$

It is important to remark that the minus sign is an intrinsic feature of the sigma operators rather than related to the effectively included spatial part of the state functions in Eq. (2.6a) and (2.6b).

(3) $s=0$, then $a_{0,0}$ remains unconstrained and there exist a set of parameters $c_{n} \in \mathbb{R}$ such that

$$
a_{0,0} \in\left\{h_{0} c_{n}\right\}_{n \in \mathbb{N}} .
$$

In general, $c_{n}$ are represented as a function of the integrals in Eq. (2.9) and therefore depend on the number of valence electrons and ions, taking part in the exchange process.

\section{TWO EXCITATIONS}

Consider two exchange bridges, $\tau=a, b$, see FIG. 1 . Let for $s=1$ the probability both electrons to be distributed over a given exchange bridges be given by the coefficients $c_{1}^{a}$ and $c_{1}^{b}$, respectively. Thus, if for a singlet state and at temperature $T_{1}$ both electrons are localized on the bridge $a$ and at temperature $T_{2}, b$ is the more energetically favorable bridge, then from Eq. (3.7) we distinguish two transitions

$$
\Delta E_{1}=\left|c_{1}^{a}\right|^{2} E_{1, M_{a}}^{a}+\left|c_{1}^{b}\right|^{2} E_{1, M_{b}}^{b}-\left|c_{0}^{a}\right|^{2} E_{0,0}^{a}
$$

and

$$
\Delta E_{2}=\left|c_{1}^{a}\right|^{2} E_{1, M_{a}}^{a}+\left|c_{1}^{b}\right|^{2} E_{1, M_{b}}^{b}-\left|c_{0}^{b}\right|^{2} E_{0,0}^{b},
$$

respectively.

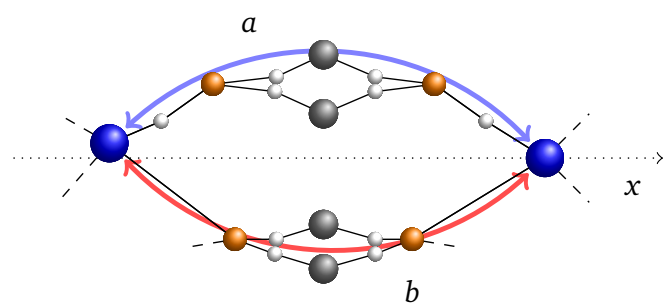

FIG. 1. Model of dimeric magnetic molecule with non symmetric exchange bridges. The effective magnetic centers are colored in blue. The upper and lower bridges are labeled by the letter $a$ and $b$, respectively. The light blue arrow depicts the bridge related with the low temperature transition, the light red arrow shows the high temperature analogue, see Eq. (5.1).

\section{A. Energy spectrum in the absence of an external magnetic field}

As the total spin commute with the Hamiltonian in Eq. (4.1), see Eq. (3.6) and (4.3), for $B=0$ we may write

$$
\hat{\mathscr{H}}|s, m\rangle=2 J a_{s, m}\left(\hat{\mathbf{s}}_{1} \cdot \hat{\mathbf{s}}_{2}\right)|s, m\rangle .
$$

In order to clarify the advantage of Eq. (5.2) we assume $T_{1}<T_{2}$ and focus on the corresponding transition energies in Eq. (5.1). Hence, with respect to the quantum numbers $s$ and $m$, from Eq. (5.2) we distinguish four energy values

$$
\frac{1}{2} J a_{1,+1}, \quad \frac{1}{2} J a_{1,0}, \quad \frac{1}{2} J a_{1,-1}, \quad-\frac{3}{2} J a_{0,0},
$$

where the first three quantities, from left to right, correspond to the triplet group and the last one is associated with the singlet state. Nevertheless, without accounting for the discrete values of the coefficients in Eq. (5.3) the excitation energy $\left|\Delta E_{2}\right|$ will remain unexplained. Therefore taking into account Eq. (4.7), (4.8), (4.9) and suggesting that $\left|\Delta E_{2}\right|<\left|\Delta E_{1}\right|$ from Eq. (5.3) we obtain an energy spectrum consisting of four levels, see FIG. 2. 


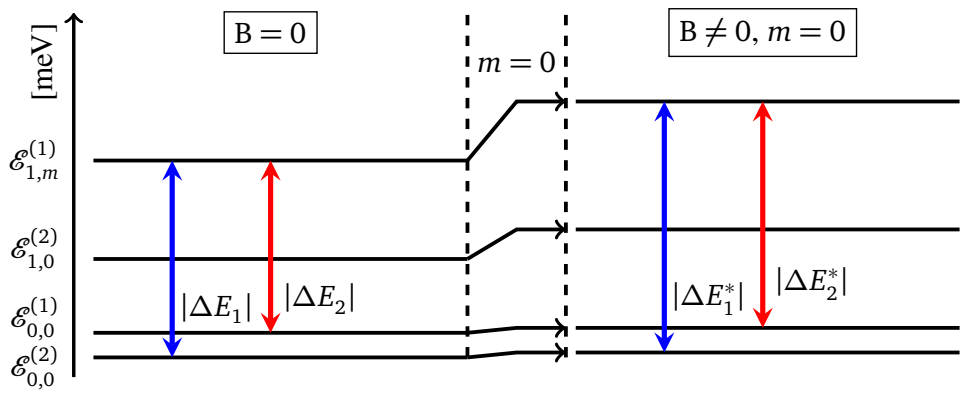

FIG. 2. Energy spectrum of the spin-half dimer obtained using the Hamiltonian in Eq. (4.1). On the left hand side the spectrum for $B=0$ is depicted. The right hand side spectrum shows the energy levels shifting due to the applied external magnetic field, where for brevity only the levels associated with the nonmagnetic states are illustrated. The blue and red arrows show both transitions, corresponding to the bridges on FIG. 1 .

Two related with the triplet and two with the singlet states. For $m=0, \pm 1$ and $n=1,2$ the set of all energy values read

$$
\mathscr{E}_{1, m}^{(1)}=\frac{1}{2} J, \quad \mathscr{E}_{1,0}^{(2)}=-\frac{1}{2} J, \quad \mathscr{E}_{0,0}^{(n)}=-\frac{3}{2} J c_{n}
$$

According to the energy conservation law and Eq. (3.7) we have

$$
\Delta E_{1}=\mathscr{E}_{0,0}^{(2)}-\mathscr{E}_{1, m}^{(1)}, \quad \Delta E_{2}=\mathscr{E}_{0,0}^{(1)}-\mathscr{E}_{1, m}^{(1)}
$$

Hence, for the model parameters we obtain

$$
J=-\frac{\Delta E_{1}}{2}, \quad c_{1}=\frac{4}{3} \frac{\Delta E_{2}}{\Delta E_{1}}-\frac{1}{3}, \quad c_{2}=1 .
$$

Depending on the type of exchange, we may obtain different values for the quantities $J$ and $c_{1}$, see Eq. (3.8) and (3.9). The value of $c_{1}$ can be determined from spectroscopic measurements.

Extracting all coefficients $a_{s, m}$ from Eq. (5.3) one can clearly distinguish the spectrum obtained from the Heisenberg model and compare it with the spectrum in Eq. (5.4). Within the framework of conventional spin bilinear Hamiltonians one assume the functions in Eq. (2.6a), (2.6b) and the coefficients in Eq. (3.7) as unique and works only with $J$ in Eq. (5.5). In such case, the exchange coupling is given by the relation

$$
J=D_{\tau}+\frac{1}{4}\left(U_{\tau}+V_{\tau}\right)+\frac{1}{4} \sqrt{16 t_{\tau}^{2}-\left(U_{\tau}-V_{\tau}\right)^{2}}
$$

and to account for the additional excitation a procedure of searching for different spin interaction terms may be started. Nevertheless, we would like to emphasize that the origin of the transition $\Delta E_{2}$ cannot be explained by the inclusion of anisotropy or higher order spin interaction terms.

\section{B. Energy spectrum with applied external magnetic field}

The applied external magnetic field affects the probability distribution of valence electrons in molecular magnets. Such phenomena can be quantitatively studied by including the parameters $h_{s}$ from Eq. (4.6) that take into account the variation of all coefficients in Eq. (2.2), (3.1) and (3.2). Therefore, considering Eq. (3.6), (5.2) and (5.4), for $B \neq 0$ we get the magnetic part of the energy spectrum

$$
\mathscr{E}_{1,+1}=\frac{1}{2} J h_{1}-g \mu_{\mathrm{B}} B, \quad \mathscr{E}_{1,-1}=\frac{1}{2} J h_{1}+g \mu_{\mathrm{B}} B,
$$

and for $n=1,2$ the nonmagnetic part

$$
\mathscr{E}_{1,0}^{(1)}=\frac{1}{2} J h_{1}, \quad \mathscr{E}_{1,0}^{(2)}=-\frac{1}{2} J h_{1}, \quad \mathscr{E}_{0,0}^{(n)}=-\frac{3}{2} J h_{0} c_{n},
$$

where we omitted the superscript in Eq. (5.6) since it has no contribution. Calculating the energy level shifting, $\Delta E_{1} \rightarrow$ $\Delta E_{1}^{*}$, and $\Delta E_{2} \rightarrow \Delta E_{2}^{*}$, we have to take into consideration only the energy levels associated with the nonmagnetic states, see FIG. 2. Thus, accounting for the energy conservation law, from Eq. (5.7) we have

$$
\Delta E_{1}^{*}=\mathscr{E}_{0,0}^{(2)}-\mathscr{E}_{1,0}^{(1)}, \quad \Delta E_{2}^{*}=\mathscr{E}_{0,0}^{(1)}-\mathscr{E}_{1,0}^{(1)}
$$

Using the relations in Eq. (5.8) together with the parameters in Eq. (5.5) for the field parameters we obtain

$$
\begin{aligned}
& h_{0}=\frac{\Delta E_{2}^{*}-\Delta E_{1}^{*}}{\Delta E_{2}-\Delta E_{1}} \\
& h_{1}=2 \frac{\Delta E_{2}^{*}+\Delta E_{1}^{*}}{\Delta E_{1}}-h_{0} \frac{2 \Delta E_{2}+\Delta E_{1}}{\Delta E_{1}} .
\end{aligned}
$$

The values of $h_{s}$ can be fixed form the magnetization and lowtemperature susceptibility measurements. Therefore, as the absolute temperature alter the values of both parameters in Eq. (5.9) in order to calculate the contribution of $B$ correctly one has to perform measurements only at very low temperatures.

\section{CONCLUSION}

We studied the role of bridging complexes in the exchange processes and evaluated their contribution within the framework of a spin-half dimer molecular magnet. To account for the influence of the intermediate structure we assume that the overall structure consists of more that one favorable exchange pathway. Within the framework of such assumptions none of 
the conventional spin models provide an appropriate energy spectrum. Therefore, to address all relevant features we proposed a formalism based on an adequate bilinear spin Hamiltonian, Eq. (4.1). It is important to emphasize that with respect to a certain representation the $\sigma$-operators are not unique.

Although the exchange Hamiltonian in Eq. (4.1) is an approximate model, it may describe reasonably well the magnetism in real compounds. Recently [28] we demonstrated the application of Eq. (4.1) in larger spin clusters. We analyzed the inelastic neutron scattering spectrum of the trimeric compounds $\mathrm{A}_{3} \mathrm{Cu}_{3}\left(\mathrm{PO}_{4}\right)_{4}, \mathrm{~A}=(\mathrm{Ca}, \mathrm{Sr}, \mathrm{Pb})$ and Nickel tetramer spin cluster $\mathrm{Ni}_{4} \mathrm{Mo}_{12}$ and we obtained results consistent with the available spectroscopic measurements [51-54].

In conclusion, we would like to point out that the existence of more than one transition at the same temperature would have been possible assuming more than two unpaired electrons and more than two magnetic centers. The probability to observe the aforementioned features increases with the size of the magnetic cluster. In the real compounds a distortion in structure's symmetry would have to be taken into account for the observation of a peculiar magnetic spectrum. Nevertheless, in compounds with only two or three distinct ions and periodic structure the discussed features cannot be observed, see Eq. (3.8). Therefore, the application of the proposed method remains restricted to a specific variety of spin clusters. For example, clusters in which the electrons are not localized around a certain ion and on the other hand are not a part of conduction band.

\section{ACKNOWLEDGMENTS}

This work was supported by the Bulgarian National Science Fund under contract DN/08/18.
[1] P. Jeffrey Hay, Jack C. Thibeault, and Roald Hoffmann, "Orbital interactions in metal dimer complexes," J. Am. Chem. Soc. 97, 4884 (1975).

[2] Timothy R. Felthouse, Edward J. Laskowski, and David N. Hendrickson, "Magnetic exchange interactions in transition metal dimers. 10. Structural and magnetic characterization of oxalate-bridged, bis(1,1,4,7,7-pentaethyldiethylene triamine)oxalatodicopper tetraphenylborate and related dimers. Effects of nonbridging ligands and counterions on exchange interactions," Inorg. Chem. 16, 1077 (1977).

[3] Jürgen Lorösch, Udo Quotschalla, and Wolfgang Haase, "Magneto-structural dependencies for asymmetrically bridged Cu(II) dimers," Inorganica Chim. Acta 131, 229 (1987).

[4] Stefan Gehring, Peter Fleischhauer, Helmut Paulus, and Wolfgang Haase, "Ferromagnetic exchange coupling and magnetostructural correlations in mixed-bridged trinuclear copper(II) complexes. Magnetic data and theoretical investigations and crystal structures of two angled CuII3 complexes," Inorg. Chem. 32, 54 (1993).

[5] Harald Astheimer and Wolfgang Haase, "Direct theoretical $a b$ initio calculations in exchange coupled copper (II) dimers: Influence of structural and chemical parameters in modeled copper dimers," J. Chem. Phys. 85, 1427 (1986).

[6] Hector W. L. Fraser, Gary S. Nichol, Gunasekaran Velmurugan, Gopalan Rajaraman, and Euan K. Brechin, "Magneto-structural correlations in a family of di-alkoxo bridged chromium dimers," Dalton Trans. 46, 7159 (2017).

[7] Marie France. Charlot, Olivier. Kahn, Max. Chaillet, and Christiane. Larrieu, "Interaction between copper(II) ions through the azido bridge: concept of spin polarization and ab initio calculations on model systems," J. Am. Chem. Soc. 108, 2574 (1986).

[8] Michael A. Aebersold, Béatrice Gillon, Olivier Plantevin, Luca Pardi, Olivier Kahn, Pierre Bergerat, Ingo von Seggern, Felix Tuczek, Lars Öhrström, André Grand, and E. Lelièvre-Berna, "Spin Density Maps in the Triplet Ground State of $\left[\mathrm{Cu}_{2}(t-\right.$ Bupy $\left.)_{4}\left(\mathrm{~N}_{3}\right)_{2}\right]\left(\mathrm{ClO}_{4}\right)_{2}(t$-Bupy=p-tert -butylpyridine $)$ : A Polarized Neutron Diffraction Study," J. Am. Chem. Soc. 120, 5238 (1998).

[9] Joan Ribas, Montserrat Monfort, Carmen Diaz, Carles Bastos, and Xavier Solans, "New antiferromagnetic dinuclear complexes of nickel(II) with two azides as bridging lig- ands. Magneto-structural correlations," Inorg. Chem. 32, 3557 (1993).

[10] Joan Ribas, Albert Escuer, Montserrat Monfort, Ramon Vicente, Roberto Cortés, Luis Lezama, and Teófilo Rojo, "Polynuclear NiII and MnII azido bridging complexes. Structural trends and magnetic behavior," Coord. Chem. Rev. 193-195, 1027 (1999).

[11] Mehul H. Sadhu, Corine Mathoniere, Yogesh P. Patil, and Sujit Baran Kumar, "Binuclear copper(II) complexes with N3scoordinate tripodal ligand and mixed azide-carboxylate bridges: Synthesis, crystal structures and magnetic properties," Polyhedron 122, 210 (2017).

[12] Xin-Hua Zhao, Lin-Dan Deng, Yan Zhou, Dong Shao, DongQing Wu, Xiao-Qin Wei, and Xin-Yi Wang, "Slow Magnetic Relaxation in One-Dimensional Azido-Bridged Co ${ }^{\mathrm{ii}} \mathrm{Com}-$ plexes," Inorg. Chem. 56, 8058 (2017).

[13] Panagiotis Angaridis, Jeff W. Kampf, and Vincent L. Pecoraro, "Multinuclear Fe(III) Complexes with Polydentate Ligands of the Family of Dicarboxyimidazoles: Nuclearity- and TopologyControlled Syntheses and Magneto-Structural Correlations," Inorg. Chem. 44, 3626 (2005).

[14] Cle Donacier Mekuimemba, Françoise Conan, Antonio J. Mota, Maria A. Palacios, Enrique Colacio, and Smail Triki, "On the Magnetic Coupling and Spin Crossover Behavior in Complexes Containing the Head-to-Tail $\left[\mathrm{Fe}_{2}^{\mathrm{ii}}(\mu-\mathrm{SCN})_{2}\right]$ Bridging Unit: A Magnetostructural Experimental and Theoretical Study," Inorg. Chem. 57, 2184 (2018).

[15] Luisa Gregoli, Chiara Danieli, Anne-Laure Barra, Petr Neugebauer, Giovanna Pellegrino, Giordano Poneti, Roberta Sessoli, and Andrea Cornia, "Magnetostructural Correlations in Tetrairon(III) Single-Molecule Magnets," Chem. Eur. J. 15, 6456 (2009).

[16] R. Viennois, E. Giannini, D. van der Marel, and R. Černý, "Effect of Fe excess on structural, magnetic and superconducting properties of single-crystalline $\mathrm{Fe}_{1+x} \mathrm{Te}_{1-y} \mathrm{Se}_{y}$, J J. Solid State Chem. 183, 769 (2010).

[17] Claudia Loose, Eliseo Ruiz, Berthold Kersting, and Jens Kortus, "Magnetic exchange interaction in triply bridged dinickel(II) complexes," Chemical Physics Letters 452, 38-43 (2008). 
[18] Anangamohan Panja, Narayan Ch. Jana, Sarmistha Adak, Paula Brandão, Lubor Dlháň, Ján Titiš, and Roman Boča, "The structure and magnetism of mono- and di-nuclear $\mathrm{Ni}(\mathrm{II})$ complexes derived from $\left\{\mathrm{N}_{3} \mathrm{O}\right\}$-donor Schiff base ligands," New J. Chem. 41, 3143 (2017).

[19] Avijit Das, Kisholoy Bhattacharya, Sanjib Giri, and Ashutosh Ghosh, "Synthesis, crystal structure and magnetic properties of a dinuclear and a trinuclear $\mathrm{Ni}(\mathrm{II})$ complexes derived from tetradentate ONNO donor Mannich base ligands," Polyhedron 134, 295 (2017).

[20] Toby J. Woods, Heather D. Stout, Brian S. Dolinar, Kuduva R. Vignesh, Maria F. Ballesteros-Rivas, Catalina Achim, and Kim R. Dunbar, "Strong Ferromagnetic Exchange Coupling Mediated by a Bridging Tetrazine Radical in a Dinuclear Nickel Complex," Inorg. Chem. 56, 12094 (2017).

[21] John B. Goodenough, "Theory of the Role of Covalence in the Perovskite-Type Manganites [La,M(II)] $\mathrm{MnO}_{3}$," Physical Review 100, 564-573 (1955).

[22] G. C. DeFotis, E. D. Remy, and C. W. Scherrer, "Magnetic and structural properties of $\mathrm{Mn}(\mathrm{SCN})_{2}\left(\mathrm{CH}_{3} \mathrm{OH}\right)_{2}$ : A quasitwo-dimensional Heisenberg antiferromagnet," Phys. Rev. B 41, 9074 (1990).

[23] Neil A. Law, Jeff W. Kampf, and Vincent L. Pecoraro, "A magneto-structural correlation between the Heisenberg constant, $J$, and the $\mathrm{Mn}-\mathrm{O}-\mathrm{Mn}$ angle in $\left[\mathrm{Mn}^{\mathrm{IV}}(\mu-\mathrm{O})\right]_{2}$ dimers," Inorganica Chim. Acta 297, 252 (2000).

[24] Myung Joon Han, Taisuke Ozaki, and Jaejun Yu, "Electronic structure, magnetic interactions, and the role of ligands in $\operatorname{Mn}_{n}(n=4,12)$ single-molecule magnets," Physical Review B 70, 184421 (2004).

[25] N.J. Perks, R.D. Johnson, C. Martin, L.C. Chapon, and P.G. Radaelli, "Magneto-orbital helices as a route to coupling magnetism and ferroelectricity in multiferroic $\mathrm{CaMn}_{7} \mathrm{O}_{12}$," Nature Communications 3 (2012).

[26] Tulika Gupta and Gopalan Rajaraman, "Modelling spin Hamiltonian parameters of molecular nanomagnets," Chemical Communications 52, 8972-9008 (2016).

[27] Mikko M. Hänninen, Antonio J. Mota, Reijo Sillanpää, Sourav Dey, Gunasekaran Velmurugan, Gopalan Rajaraman, and Enrique Colacio, "Magneto-Structural Properties and Theoretical Studies of a Family of Simple Heterodinuclear Phenoxide/Alkoxide Bridged $\mathrm{Mn}^{\text {iii }} \mathrm{Ln}^{\text {iii }}$ Complexes: On the Nature of the Magnetic Exchange and Magnetic Anisotropy," Inorg. Chem. 57, 3683 (2018).

[28] M. Georgiev and H. Chamati, "A systematic approach to determine the spectral characteristics of molecular magnets," arXiv:1805.01382 [cond-mat] (2018).

[29] Ian Fleming, Molecular Orbitals and Organic Chemical Reactions (Wiley, Chichester, UK, 2009).

[30] J. E. Lennard-Jones, "The electronic structure of some diatomic molecules," Trans. Faraday Soc., 25, 668 (1929).

[31] Robert S. Mulliken, "Intensities of Electronic Transitions in Molecular Spectra II. Charge-Transfer Spectra," J. Chem. Phys. 7, 20 (1939).

[32] J. Lennard-Jones, "The Molecular Orbital Theory of Chemical Valency. I. The Determination of Molecular Orbitals," Proc. R. Soc. London, Ser. A 198, 1 (1949).

[33] J. A. Pople, D. P. Santry, and G. A. Segal, "Approximate SelfConsistent Molecular Orbital Theory. I. Invariant Procedures," J. Chem. Phys. 43, S129 (1965).

[34] J. A. Pople and G. A. Segal, "Approximate Self-Consistent Molecular Orbital Theory. III. CNDO Results for $\mathrm{AB}_{2}$ and $\mathrm{AB}_{3}$ Systems," J. Chem. Phys. 44, 3289 (1966).
[35] J. A. Pople, D. L. Beveridge, and P. A. Dobosh, "Approximate Self-Consistent Molecular-Orbital Theory. V. Intermediate Neglect of Differential Overlap," J. Chem. Phys. 47, 2026 (1967).

[36] Walter England, Lydia S. Salmon, and Klaus Ruedenberg, "Localized molecular orbitals: A bridge between chemical intuition and molecular quantum mechanics," in Molecular Orbitals, Vol. 23/1 (Springer-Verlag, Berlin/Heidelberg, 1971) p. 31.

[37] Alan E. Reed and Frank Weinhold, "Natural localized molecular orbitals," J. Chem. Phys. 83, 1736 (1985).

[38] J. C. Slater, "The Theory of Complex Spectra," Phys. Rev. 34, 1293 (1929).

[39] J. C. Slater, "Note on Hartree's Method," Phys. Rev. 35, 210 (1930).

[40] D. R. Hartree, "The Wave Mechanics of an Atom with a nonCoulomb Central Field. Part III. Term Values and Intensities in Series in Optical Spectra," Math. Proc. Camb. Philos. Soc. 24, 426 (1928).

[41] Takao Tsuneda, "Hartree-Fock Method," in Density Functional Theory in Quantum Chemistry (Springer Japan, Tokyo, 2014) p. 35.

[42] P. W. Anderson, "Antiferromagnetism. Theory of Superexchange Interaction," Phys. Rev. 79, 350 (1950).

[43] Junjiro Kanamori, "Superexchange interaction and symmetry properties of electron orbitals," J. Phys. Chem. Solids 10, 87 (1959).

[44] P. W. Anderson, "New Approach to the Theory of Superexchange Interactions," Phys. Rev. 115, 2 (1959).

[45] W. Heisenberg, "Mehrkörperproblem und Resonanz in der Quantenmechanik," Zeitschrift für Physik 38, 411 (1926).

[46] J. Hubbard, "Electron correlations in narrow energy bands," Proc. R. Soc. A 276, 238-257 (1963).

[47] J. Hubbard, "Electron Correlations in Narrow Energy Bands. III. An Improved Solution," Proc. R. Soc. A 281, 401-419 (1964).

[48] Achim Müller, Christian Beugholt, Paul Kögerler, Hartmut Bögge, Sergey Bud'ko, and Marshall Luban, " $\left[\mathrm{Mo}_{12}^{\mathrm{V}} \mathrm{O}_{30}\left(\mu_{2^{-}}\right.\right.$ $\mathrm{OH})_{10} \mathrm{H}_{2}\left\{\mathrm{Ni}^{\mathrm{II}}\left(\mathrm{H}_{2} \mathrm{O}\right)_{3}\right\}_{4}$ ], a Highly Symmetrical $\varepsilon$-Keggin Unit Capped with Four $\mathrm{Ni}^{\mathrm{II}}$ Centers: Synthesis and Magnetism," Inorg. Chem. 39, 5176 (2000).

[49] Nicola Marzari, Arash A. Mostofi, Jonathan R. Yates, Ivo Souza, and David Vanderbilt, "Maximally localized Wannier functions: Theory and applications," Rev. Mod. Phys. 84, 1419 (2012).

[50] Philip W. Anderson, "Theory of Magnetic Exchange Interactions:Exchange in Insulators and Semiconductors," in Solid State Physics, Vol. 14 (Elsevier, 1963) p. 99.

[51] M. Matsuda, K. Kakurai, A. A. Belik, M. Azuma, M. Takano, and M. Fujita, "Magnetic excitations from the linear Heisenberg antiferromagnetic spin trimer system $\mathrm{A}_{3} \mathrm{Cu}_{3}\left(\mathrm{PO}_{4}\right)_{4}(\mathrm{~A}=\mathrm{Ca}, \mathrm{Sr}$, and Pb)," Phys. Rev. B 71, 144411 (2005).

[52] A. Podlesnyak, V. Pomjakushin, E. Pomjakushina, K. Conder, and A. Furrer, "Magnetic excitations in the spin-trimer compounds $\mathrm{Ca}_{3} \mathrm{Cu}_{3-x} \mathrm{Ni}_{x}\left(\mathrm{PO}_{4}\right)_{4}(x=0,1,2)$," Phys. Rev. B 76, 064420 (2007).

[53] J. Nehrkorn, M. Höck, M. Brüger, H. Mutka, J. Schnack, and O. Waldmann, "Inelastic neutron scattering study and Hubbard model description of the antiferromagnetic tetrahedral molecule $\mathrm{Ni}_{4} \mathrm{Mo}_{12}$," Eur. Phys. J. B 73, 515 (2010).

[54] A. Furrer, K. W. Krämer, Th. Strässle, D. Biner, J. Hauser, and H. U. Güdel, "Magnetic and neutron spectroscopic properties of the tetrameric nickel compound $\left[\mathrm{Mo}_{12} \mathrm{O}_{28}\left(\mu_{2}-\mathrm{OH}\right)_{9}\left(\mu_{2^{-}}\right.\right.$ $\left.\mathrm{OH})_{3}\left\{\mathrm{Ni}\left(\mathrm{H}_{2} \mathrm{O}\right)_{3}\right\}_{4}\right] \cdot 13 \mathrm{H}_{2} \mathrm{O}$," Phys. Rev. B 81, 214437 (2010). 